\title{
Psychological State Diagnosis using Deep Learning Techniques
}

\author{
Chandan A, Ajay Umakanth, Adarsh N, Dr. Girijamma H A.
}

\begin{abstract}
Psychological State or Depression is a looming mental health problem in the society. This, negatively affects many families, relationships, jobs. But to provide effective treatment, there is no awareness about this. Most people do not give much thought to this as they do to physical problems due to reasons which include that they are shy, afraid or negligent about this. A feasible solution to this is to create awareness about this so that people can actively seek out help and just not choose to suffer in silence. This paper proposes an approach to detect psychological state or depression in people using mainly non-verbal and involuntary cues with the help of a standard questionnaire. The subject wears the MindWave device by NeuroSky and pairs it with a smartphone. Then a standard questionnaire is answered during which the data on brain waves and emotions are collected simultaneously by MindWave and the smartphone camera respectively. The data collected is then used to train a model that will give a score pertaining to the severity of depression in a person, thus aiming to give a better accuracy compared to all the devices present.
\end{abstract}

Keywords: Brainwaves, Depression Detection, Diagnosis, Emotion, NeuroSky, PHQ-9

\section{INTRODUCTION}

Depression or psychological state is one of the mental health disorders that is colloquial and serious illness which negatively affects significant impairments in daily life. Every two in three who have been diagnosed with a mental disorder do not seek help from an expert. The World Health Organization (WHO) tells that stigma, discrimination, and negligence are the reasons for proper care and treatment not reaching people with mental disorders [1]. The remaining one-third of people have no idea of what is going on in their lives and end up having suicidal thoughts. Anhedonia or the loss of interest in various activities that usually bring pleasure is one major symptom. The reasons for getting depressed vary from unemployment, family issues, work pressure, etc. Over 8 lakh people die every year due to suicide and depression is one of the prime reasons for it. At some point of their life, quarter of the entire population will be affected.

Revised Manuscript Received on December 15, 2019.

Adarsh N, CSE department, RNSIT, Bangalore, India. Email: adarshnagraj43@gmail.com

Ajay Umakanth, CSE department, RNSIT, Bangalore, India. Email: ajayunp@gmail.com

Chandan A, CSE department, RNSIT, Bangalore, India. Email: chandan190997@gmail.com

Dr. Girijamma H A, CSE department, Professor RNSIT, Bangalore, India. Email: girijakasal@gmail.com
Treatment is available in the following category:

1) Support groups, where people with common concerns and bad experiences gather together and talk to each other providing comforts, moral support, and advice through their struggles.

2) Psychotherapy, where the people having mental health issues consult a psychotherapist and this psychotherapist treats them psychologically rather than medical means.

3) Anti-depressant pills, where people get treatment for their mental health issues through medication.

There are new ways to fight this disorder but these are not efficient enough due to lack of resources and proper healthcare providers [2]. In the diagnostics and statically manual are listed some conditions which should test positive for a person to be diagnosed with clinical depression [3]. But these techniques do not include visual indicators, involuntary cues, etc. that are good indicators of depression. Even though there's a way to get treatment, people neglect it as it is not physical or they hesitate to expose their issues to the world. Hence, giving way for the following proposed system.

The proposed system considers X-factors such as emotion, brain waves, standard PHQ-9 questionnaires on a subject to detect depression or psychological state which is more efficient than the existing system. This is due to the fact that it considers involuntary components that makes it immune to false and unclear answers by the subject. Any user can take this test on their mobile app after pairing the NeuroSky device through Bluetooth, making the system portable and accessible by anyone. This system is the first step in faster and efficient diagnosis thus helping combat depression.

\section{LITERATURE SURVEY}

Multinomial Naive Bayes and Support Vector Machine methods were used by [4] which involves usage of the Natural Language Techniques. The data collected are from public Twitter accounts who have stated that they have been through depression. The parameters used for the processing of the data are tokenization, stemming, stop word removal, POS tagger (Part of Speech) which identifies the essential words. The proposed system yields an accuracy of $83 \%$ using Multinomial Naive Bayes and 79\% in the case of SVM.

[5] tells us about PHQ questionnaires that are self-administered and have a sublime diagnostic accuracy collating to the PRIME-MD administered clinically. PHQ9 questionnaires are widely used in research and clinical settings. Patients are classified by PHQ9 into three classes: Clinical, dysthymia and no depression. 


\section{Psychological State Diagnosis using Deep Learning Techniques}

[6] makes use of facial expression to analyze the EEG signal, using facial and EEG method emotions of the subject are detected. Continuous Conditional Random Fields model, which is a probabilistic model used to classify the sequential data. MAHNOB-HCI database was used for emotion detection. Samples which are obtained from the EEG are averaged so as to reduce the signal to noise ratio. The supervised descent method (SDM) uses 49 facial fiducial points to track the facial expression. Long-Short-Term Memory Recurrent Neural Networks (LSTM-RNN) are used for emotion recognition. To observe the EEG attributes using facial expressions linear mixed-effect model was used.

In [7], EEG signals are used to detect the facial expression of the subject. For extracting the salient features, two methods were adopted: Fractal Analysis and Interval Analysis. Daubechies wavelet function (db4) for extracting the statistical features. Fuzzy C-Means (FCM) is used to cluster data into four different categories: happy, sad, surprised and disgusted. The accuracy of this approach will depend on the extent of electrode-scalp contact, movement and Vascular (ECG), Ocular (EoG), Gloss kinetic and Muscular (EMG) artifacts.

The paper [8] makes use of the Local tetra pattern and Fisher vector algorithms. For description and representation of an image, Gaussian mixture model (GMM) is used. Local tetra pattern is used for feature extraction. The facial samples obtained from the AVEC database are preprocessed to obtain an area of interest. In the image preprocessing stage, the image is converted to grayscale, then resized and some morphological operations are carried out. Fisher vector will classify the image and encode. K-Nearest Neighbor Algorithm identifies and classifies them into depressed and not depressed category. The proposed accuracy is $87 \%$. The depressed person with a happier face may not be recognized. The approach [9] uses XA-BiLSTM , a deep learning model for depression detection. It consists of two essential entities: XGBoost and Attention-BiLSTM neural network. Data filtration is done by the XGBoost, XGBoost will directly output the negative samples and the positive samples are passed on to Attention-BiLSTM. Backward and forward sequences are fed to the learning algorithm to get the representation of the word. A normalized weight for each word was obtained through a softmax function. The accuracy increased from 16.6 to 17.6 when compared to other models like Bow-SVM, Bow-MNB and User model-CNN.

In [10], a semantic graph is used to correlate depression and its symptoms. natural processing techniques and hybrid statistical analysis are used to build the semantic-graph. To build depression symptoms, the important entities from the twitter data are extracted and sent to Automatic-Extract Keyword for Specific Term (AEKW) algorithm that combines Word2Vec, RAKE and cooccurrence. Entities obtained are combined to form a matrix. For each depression entity, the AEKW algorithm calculates scores and assigned. [11] uses data from Shared Task organization. Bag of Words approach. Four different binary classifiers are used to identify the likelihood of depression, a Logistic regression classifier, Linear Support Vector Classifier, Naive Bayes algorithm and Decision Trees are used to classify. Unigram-based Naive

Bayes approach has an accuracy of $86 \%$, which is the highest accuracy attained when compared to the rest of the classifiers. All the above-mentioned systems make use of many important factors, but not all at once. Depression in detection is based on many factors including verbal, non-verbal and involuntary cues. If we choose to not include even one of them, the accuracy of any model should decrease. The proposed system takes into consideration all the above factors so that the accuracy and robustness of depression detection are good.

\section{IMPLEMENTATION}

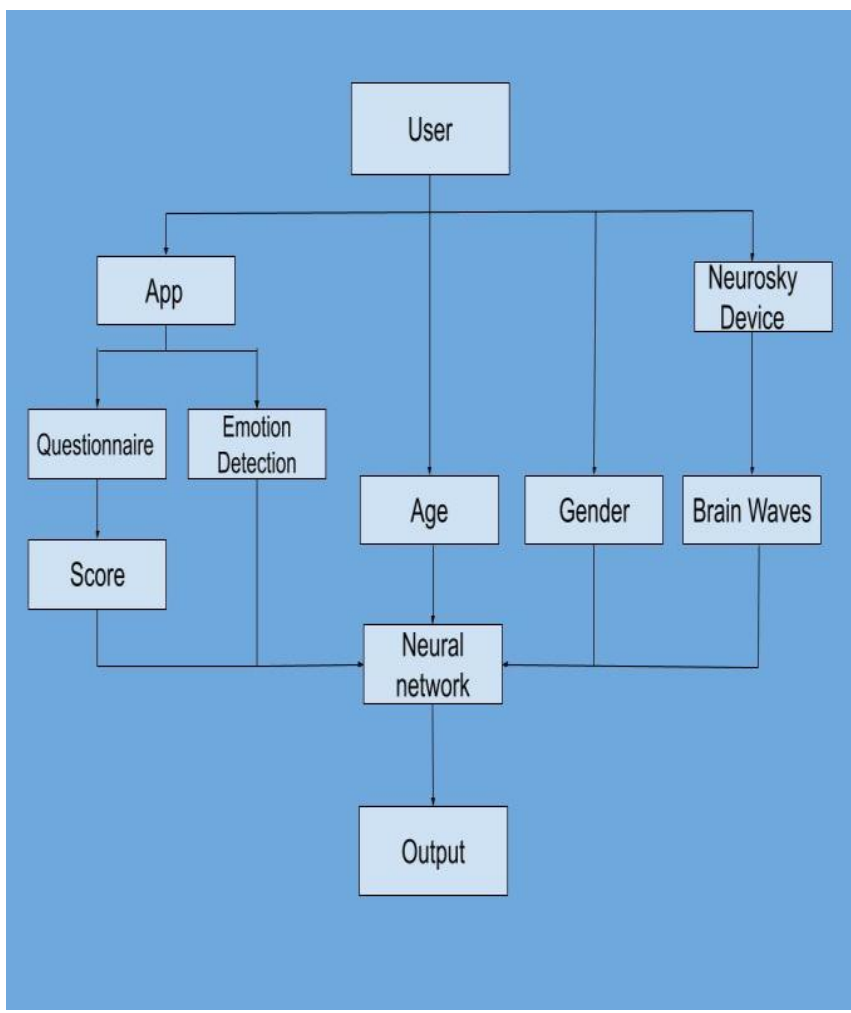

Fig 3.1 - Proposed System

Implementation of the proposed model includes various steps and methods. The method makes use of the brain waves generated from the NeuroSky device, a standard questionnaire created by many psychiatrists and emotion detection (Fig 3.1). The user is subjected to the questionnaires, during which facial emotions and the brain waves are detected and all these data are collected. The collected data is sent to deep learning model This proposed system should give a high accuracy as many methods are being used. The data collected from the user are mainly involuntary that should give good accuracy as these are immune to false answers, unclear answers, etc. Using this, the model tries to predict the severity of depression in the individual with good accuracy considering factors that just the questionnaire would fail to answer.

\section{A. Standard Questionnaire}

Patient Health Questionnaires (PHQ-9)

PHQ-9 consisting of 9 questions, is a self-administered questionnaire. These questions give out a score between 0-25 which indicates the severity of a person's depression. 
Each question is a multiple-choice question consisting of 4 choices. Each choice has an associated score, which is added to reveal the final score. Table I shows the questions in PHQ-9

PHQ-9 is chosen for this model as it is a very small yet accurate questionnaire. Various studies have shown that this questionnaire has a high specificity, sensitivity, and accuracy [12].

Table I - Details of PHQ-9

\begin{tabular}{|c|c|c|c|c|}
\hline $\begin{array}{l}\text { Over the last } 2 \text { wecks, how often have you beci } \\
\text { bothered by any of the following problems? }\end{array}$ & $\begin{array}{l}\text { Not at } \\
\text { all }\end{array}$ & $\begin{array}{c}\text { Several } \\
\text { Days }\end{array}$ & $\begin{array}{l}\text { More } \\
\text { than } \\
\text { half the } \\
\text { days }\end{array}$ & $\begin{array}{l}\text { Nearly } \\
\text { extry } \\
\text { day }\end{array}$ \\
\hline 1. Little interest or pleasure in doing things & 0 & 1 & 2 & 3 \\
\hline 2. Feeling down, depeesed, of hopesless & 0 & 1 & 2 & 3 \\
\hline $\begin{array}{l}\text { 3. Trouble falling or staying aslepp, or sleeping too } \\
\text { mach }\end{array}$ & 0 & 1 & 2 & 3 \\
\hline 4. Fecling tired or having little energy & 0 & 1 & 2 & 3 \\
\hline S. Poor appetile of onereating & 0 & 1 & 2 & 3 \\
\hline $\begin{array}{l}\text { 6. Feeling bad about yourself - } \alpha \text { that you ace a failure } \\
\text { os have let yourelf of your family doent }\end{array}$ & 0 & 1 & 2 & 3 \\
\hline $\begin{array}{l}\text { 7. Trouble concentrating on things, such as rading the } \\
\text { scuspaper of wathing telovision }\end{array}$ & 0 & 1 & 2 & 3 \\
\hline $\begin{array}{l}\text { 8. Moving of speaking so slywly that other poople } \\
\text { could have noticed. Or the opposite - being so fidgety } \\
\text { of restles that you have bren moving around a lor moes } \\
\text { than uswal }\end{array}$ & 0 & 1 & 2 & 3 \\
\hline $\begin{array}{l}\text { 9. Thooghts that you would be better off dead, of of } \\
\text { burting yourself in some way }\end{array}$ & 0 & 1 & 2 & 3 \\
\hline
\end{tabular}

Total Score: 14 Minimal depression; S.9 Mild depresion; 10-14 Moderate depresiban; 15-19 Moderately seere depresion; 20-27 Severe depression

\section{B. MindWave Mobile: by NeuroSky Device}

This is a small, simple and portable device that is worn by the user on the head. The EEG power spectrum is discerned by the kit. The brain consists of many neurons that are constantly exchanging ions between them. When a significant number of these likely charged ions leave many neurons in a region, these can push their neighbors, which pushes their neighbors and so on. Thus, they generate a wave in the brain. These ions can then interact with the electrodes in the device, causing a reading. Different frequencies of waves are generated based on the different activities being performed. All the different waves and their frequencies are shown in Table II.

Table II - Different types of brain waves

\begin{tabular}{|c|c|l|}
\hline Name & $\begin{array}{c}\text { Frequency } \\
(\mathbf{H z})\end{array}$ & \multicolumn{1}{|c|}{ Description } \\
\hline$\alpha$-waves & $7.9-11.9$ & Resting-State \\
\hline$\beta$-waves & $11.9-37.9$ & Normal Consciousness \\
\hline$\gamma$-waves & $37.9-41.5$ & Rapid information relay \\
\hline
\end{tabular}

This device is worn by the user while interacting with the mobile phone. The person's brain waves are read during the answering of each question. Then these waves are fed into the neural network along with other inputs to diagnose the person's severity of depression.

\section{Emotion Detection}

Emotions play a huge role in non-verbal communication in human beings. These emotions are provoked in the inner part of the brain. The emotions in a person reveals many behavioral tendencies. Each person's emotions are different and the way they react to different things is also different. Each emotion in a person is accompanied by a physiological pattern that may be influenced by various traits like gender, age, ethnicity, social class, etc. So, the data regarding emotions can be of great significance for insight into a person. While answering the questionnaire, the front camera of the user's phone records the user's face. The emotion of the user on reading and answering a question is considered to be a piece of vital information. Frames in regular intervals are selected and sent to a model for emotion detection. This model first detects the face, pre-processes it and then is sent to a trained $\mathrm{CNN}$ which gives the emotion of the user at the current state. This emotion is then sent to the proposed model for emotion detection. The CNN detects the following emotions
- Angry
- Happy
- $\mathrm{Sad}$
- Disgust
- Surprised
- Neutral
- Scared

\section{Deep Learning}

Deep structured learning commonly known as deep learning, which is a crucial part of machine learning-based artificial neural networks. The term deep refers to the number of times the input gets transformed. In deep learning, each level learns to construct a better representation of the data. Multi-class Artificial- Neural- Network is used to predict the output of all the above factors. The model consists of an input layer, 3 hidden layers, and 1 output layer. The input layers take in multiple inputs, $\alpha$ - waves, $\beta$ - waves, $\delta$ - waves, $\gamma$ - waves, $\theta$ waves, age, gender, emotion, and PHQ-9 question number. The output layer gives an output between 0 to 3 that corresponds to the answer score given by the PHQ-9 questionnaire for each question. This output is fed back to the neural network as feedback. 


\section{Psychological State Diagnosis using Deep Learning Techniques}

Next, the score given by the model is averaged with the score of the questionnaire which gives us the final result. This method will be more efficient as it considers many other factors including involuntary and non-verbal cues to detect depression more accurately than a person's answer to a questionnaire. Fig 3.2 shows the complete architecture of the neural network.

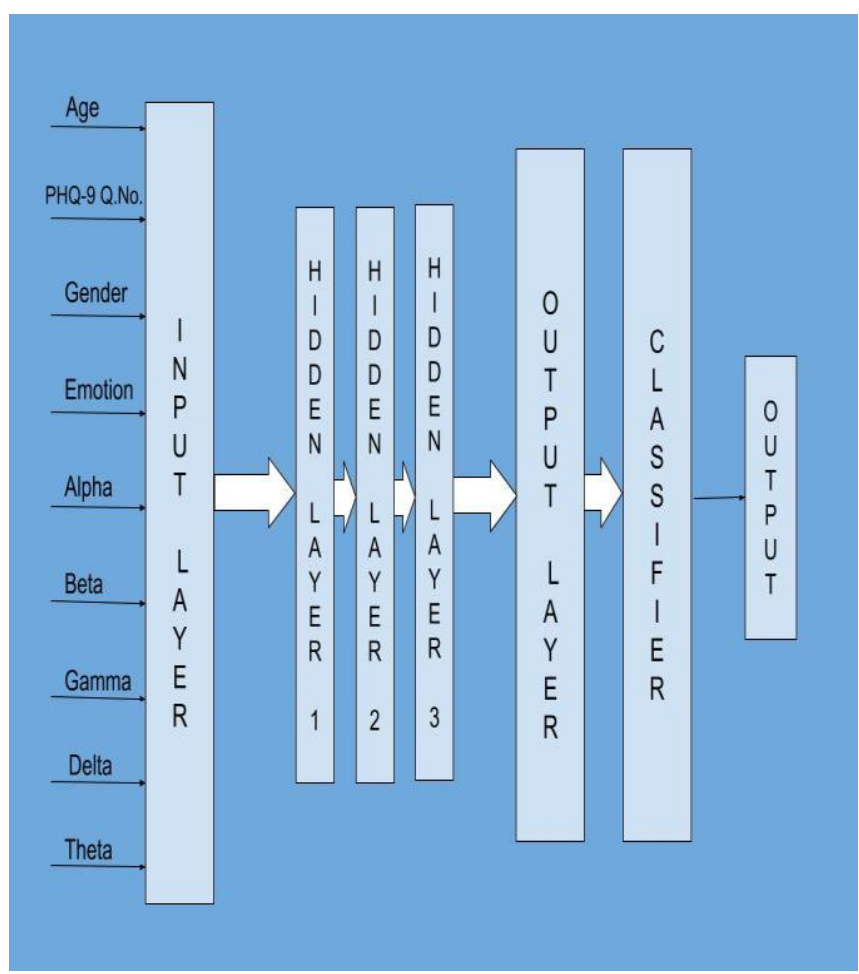

Fig 3.2 - Neural Network Architecture

\section{CONCLUSION}

Diagnosis of depression using NeuroSky MindWave device, PHQ-9 questionnaire, and their emotional response is proposed. This paper attempts to detect depression using mainly involuntary cues that are exhibited by a person. Existing systems detect depression mainly using the response of the subject. This system depends on the existing data of answers given to the PHQ-9 questionnaire. So, enough care should be given for the proper collection of data to get an accurate model.

\section{REFERENCES}

1) UN health agency reports depression now 'leading cause of disability worldwide'". UN News. 23 February 2017. Retrieved 27 June 2019

2) J. C. Mundt, P. J. Snyder, M. S. Cannizzaro, K. Chappie, and D. S. Geralt's, "Voice acoustic measures of depression severity and treatment response collected via interactive voice response (IVR) technology," Journal of neurolinguistics, vol. 20, no. 1, pp. 50-64, 2007

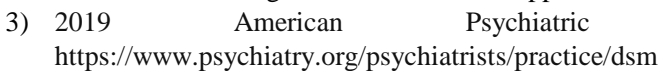

4) Deshpande, Mandar, and Vignesh Rao. "Depression detection using emotion artificial intelligence." In 2017 International Conference on Intelligent Sustainable Systems (ICISS), pp. 858-862. IEEE, 2017.

5) Kroenke, Kurt, and Robert L. Spitzer. "The PHQ-9: a new depression diagnostic and severity measure." Psychiatric annals 32, no. 9 (2002): 509-515

6) Soleymani, Mohammad, Sadjad Asghari-Esfeden, Yun Fu, and Maja Pantic. "Analysis of EEG signals and facial expressions for continuous emotion detection." IEEE Transactions on Affective Computing 7, no. 1 (2015): 17-28
7) Murugappan, M., M. Rizon, R. Nagarajan, and Sazali Yaacob. "Inferring of human emotional states using multichannel EEG." European Journal of Scientific Research 48, no. 2 (2010): 281-299

8) Kulkarni, Prajakta Bhalchandra, and Minakshee M. Patil. "Clinical Depression Detection in Adolescent by Face." In 2018 International Conference on Smart City and Emerging Technology (ICSCET), pp. 1-4. IEEE, 2018

9) Cong, Qing, Zhiyong Feng, Fang Li, Yang Xiang, Guozheng Rao, and Cui Tao. "XA-BiLSTM: a Deep Learning Approach for Depression Detection in Imbalanced Data." In 2018 IEEE International Conference on Bioinformatics and Biomedicine (BIBM), pp. 1624-1627. IEEE, 2018

10) Ma, Long, and Yan Wang. "Constructing a Semantic Graph with Depression Symptoms Extraction from Twitter." In 2019 IEEE Conference on Computational Intelligence in Bioinformatics and Computational Biology (CIBCB), pp. 1-5. IEEE, 2019

11) Nadeem, Moin. "Identifying depression on Twitter." arXiv preprint arXiv:1607.07384 (2016)

12) The PHQ-9: validity of a brief depression severity measure https://www.ncbi.nlm.nih.gov/m/pubmed/ 11556941

\section{AUTHORS PROFILE}

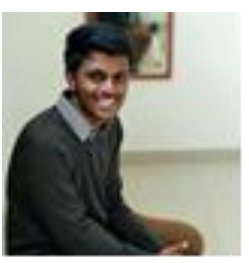

Adarsh N - Completed his Bachelor of Engineering degree in Computer Science at RNSIT. His areas of focus are Computer vision, web development.

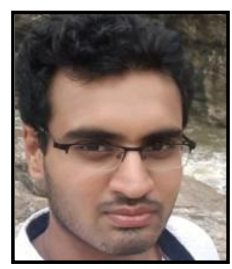

Ajay Umakanth - Completed his Bachelor of Engineering degree in Computer Science at RNSIT. His area of interests are Computer vision, web development, IOT

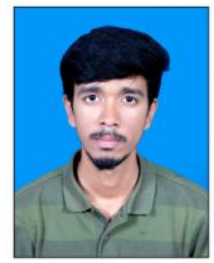

Chandan A - Completed Bachelor of Engineering degree in Computer Science at RNSIT. His research interests include Computer vision, web development.

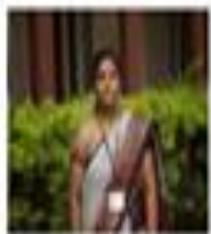

Dr. Girijamma H A-Currently working as a Prof. of Computer Science and Engineering at RNSIT, Bangalore and having experience of 26 years in teaching. Automata, compilers, data analytics, software engineering, image processing, natural language processing, and Machine learning are her areas of expertise. 